\title{
The Prevalence of Restless Legs Syndrome in Patients with Migraine: A Systematic Review and Meta-Analysis
}

\author{
Hooman Ghasemi ${ }^{1 D},{ }^{1}$ Behnam Khaledi-Paveh, ${ }^{2}$ Alireza Abdi, ${ }^{1}$ Rostam Jalali, ${ }^{1}$ \\ Nader Salari, ${ }^{1}$ Aliakbar Vaisi-Raygani, ${ }^{1}$ and Masoud Mohammadi ${ }^{1}{ }^{1}$ \\ ${ }^{1}$ Department of Nursing, School of Nursing and Midwifery, Kermanshah University of Medical Sciences, Kermanshah, Iran \\ ${ }^{2}$ Sleep Disorders Research Center, Kermanshah University of Medical Sciences, Kermanshah, Iran \\ Correspondence should be addressed to Masoud Mohammadi; masoud.mohammadi1989@yahoo.com
}

Received 12 May 2020; Revised 6 July 2020; Accepted 21 August 2020; Published 29 August 2020

Academic Editor: Filippo Brighina

Copyright (C) 2020 Hooman Ghasemi et al. This is an open access article distributed under the Creative Commons Attribution License, which permits unrestricted use, distribution, and reproduction in any medium, provided the original work is properly cited.

\begin{abstract}
Background. Migraine is a severe and debilitating neurologic disorder and is claimed to be the sixth disabling illness in the world. This study aimed to determine the overall prevalence of restless legs syndrome (RLS) in patients with migraine through a systematic review and meta-analysis. Methods. To identify and select related studies, the Scopus, ScienceDirect, Embase, SID, IranDoc, Web of Science, Knowledge Network System Medline (PubMed), and Google Scholar databases were searched. All related cross-sectional studies, published in English or Persian language between January 2000 and December 2019 and using the keywords such as migraine, restless leg syndrome, sleep disorder, RLS, and migraine disorder, were collected. The heterogeneity of the studies was assessed using the $I^{2}$ index, and the data analysis was performed using the Comprehensive Meta-Analysis software. Results. Analysis was conducted on the reported results of the final 12 articles with the total sample size of 15196 . The overall prevalence of RLS in patients with migraine was $16.3 \%$ (95\% CI: 12.6-20.8\%). The prevalence of RLS migraine patients decreased with increasing the sample size, and the prevalence of RLS migraine patients increased with increasing the research year, which was statistically significant $(P<0.05)$. Conclusion. This study highlights that RLS is high in patients with migraine, and therefore, the clinicians should be aware of its incidence and take preventive measures.
\end{abstract}

\section{Background}

Migraine is a severe and debilitating neurologic disorder which is realised with local throbbing sensation in the head. It is a type of disturbance in the subcortical aminergic sensory modulatory systems [1-3]. In other words, the disorder is a type of brain disturbance of the subcortical aminergic sensory modulatory systems [4]. Although migraine is widespread, the causes of the disorder are not well understood, and in fact, the triggers of migraine vary from a person to another. Nevertheless, many factors such as food, barometric pressure changes, and stress can cause migraines [5]. Migraine affects the quality of life and results in healthcare costs for the patients [6].

The prevalence of migraine has been reported to be ranging from 10 to $18 \%$ in some studies. The estimated prevalence of migraine is $14.9 \%$ in European countries. In addition, migraine is reported more commonly in women than men [7-9].

Restless legs syndrome (RLS) is a movement disorder in which there is a feeling of distress and a tendency to move the legs, and in some cases the arms and the neck [10]. Although the cause of RLS in unknown, it is thought that iron deficiency in brain causes primary RLS, whereas kidney failure, pregnancy, anemia, and genetics are known to be associated with secondary RLS [11]. It occurs most often at nights, and creates sleep disturbances [12].

Migraine and RLS put a heavy burden on society [13]. These two disorders have a high impact on the quality of life. Moreover, the prevalence of RLS is higher in patients with migraine than in a healthy population [14]. Some existing studies have reported an unalike prevalence of RLS in 
patients with migraine. Therefore, since intervention studies on reducing the incidence of RLS in patients with migraine require accurate and consistent information to prevent the complications of RLS, an overall prevalence of RLS in migraine patients has not been reported. Therefore, this piece of research attempts to answer the following research question: what is the overall prevalence of RLS in patients with migraine?

\section{Methods}

2.1. Search Method. Multiple databases, including ScienceDirect, Embase, Scopus, SID, IranDoc, Medline (PubMed), and Google Scholar were searched. All related cross-sectional studies published in English or Persian language, between January 2000 and December 2019, were identified and selected. The keywords used in the search process were migraine, migraineurs, restless legs syndrome, sleep disorder, RLS, and migraine disorder. Review papers, case-control, cohort, and intervention studies were excluded from the list of articles.

Moreover, the Google Scholar search engine was searched using both Persian and English keywords. AND and OR operators were added to the keywords combinations with a view to provide a more comprehensive access to all articles. For instance, the OR operator was used to examine the common names for disorders such as (migraine OR migraine headache), (sleep disorder OR sleep wake disorder), and (restless legs syndrome OR RLS), whereas, the AND operator was added in between the keywords (AND) considering the Medical Subject Headings (MeSH).

2.2. Evaluation of Articles. After collecting the relevant articles, a list of abstracts was prepared. The author(s) and journal names were redacted, yet allowing reviewers to select the full-text articles. Each article was independently examined by two reviewers, and if an article was rejected, the reason for this was mentioned. In the case of disagreement between the two reviewers, the article was then reviewed by a third reviewer. Furthermore, to ensure the comprehensiveness of the search process, the Google Scholar search engine was searched to identify any related grey literature.

The search process was conducted in accordance with the four-step PRISMA 2009 guidelines. The steps include article identification, screening, eligibility evaluation, and finally including the final studies for meta-analysis (Figure 1).

2.3. Quality Assessment and Statistical Analysis. The STROBE checklist was used to assess the quality of the studies. The total number of scores that can be obtained using the checklist is 32 . Articles with a score below 14 were considered as low-quality and were, therefore, excluded from the study.

In each study, the prevalence of RLS in patients with migraine was reported. The heterogeneity of the studies was assessed using the $I^{2}$ test. Data were analyzed using the Comprehensive Meta-Analysis software (Biostat,

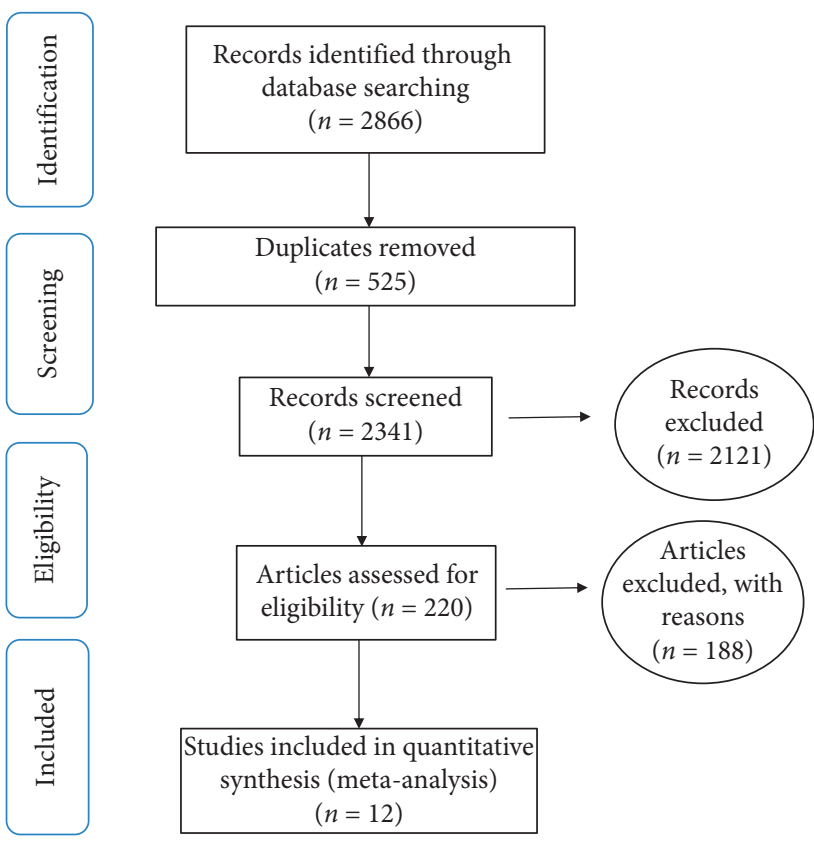

FIgUre 1: The flowchart of the stages of studies including in the systematic review and meta-analysis (PRISMA 2009).

Englewood, NJ, USA version 3). The possibility of publication bias was assessed using the funnel plots and Egger's test with the significance level of 0.05 . Moreover, in order to investigate the impacts of other potential factors on the heterogeneity of studies, the meta-regression test was used in the two factors of "sample size" and "year of study."

\section{Results}

Initially, a total of 2,866 articles were identified, of which 254 articles were obtained from IranDoc, 4 articles from SID, 402 articles from Medline (PubMed), 242 articles from ScienceDirect, 378 articles from Scopus, 526 articles from Embase, 266 articles from Web of Science, and 756 articles were obtained from Google Scholar.

During the primary reviews, 525 duplicate articles were omitted; 2,121 articles were removed during the secondary review since they were unrelated to the study subject. Additionally, 188 articles were excluded due to either the lack of access to the abstract and the original article or their lowquality, and ultimately 12 articles were included in the metaanalysis (Table 1).

3.1. Investigation of Heterogeneity and Publication Bias. The heterogeneity of the studies was evaluated using the $I^{2}$ test; $I^{2}=96.8 \%$ was obtained, indicating a high heterogeneity among the included studies. Therefore, the random effects model was used to combine the results of the studies together. Furthermore, the possibility of publication bias in the studies was assessed using Egger's test (Figure 2). The publication bias was not statistically significant $(P=0.867)$.

The total sample size from all collected studies was 15,196 . Based on the meta-analysis performed in this study, 
TABLE 1: Studies included in the meta-analysis.

\begin{tabular}{|c|c|c|c|c|c|}
\hline Study & Year published & Location & Age & Sample size & Prevalence \\
\hline Calhoun et al. [15] & 2006 & North Carolina & $39.47 \pm 11.32$ & 147 & 23.8 \\
\hline Chen et al. [16] & 2010 & Taipei & $42.1 \pm 13.3$ & 772 & 11.3 \\
\hline Chen et al. [17] & 2015 & Taiwan & - & 403 & 9.3 \\
\hline Cho et al. [18] & 2015 & Korea & - & 143 & 9.09 \\
\hline Cologno et al. [19] & 2008 & Italy & $37.1 \pm 10.8$ & 164 & 26 \\
\hline d'Onofrio et al. [20] & 2011 & Italy & $38.2 \pm 14.1$ & 63 & 9.52 \\
\hline Muayqil et al. [21] & 2018 & Saudi Arabia & $30.99 \pm 10.76$ & 1330 & 29.2 \\
\hline Ødegård et al. [22] & 2010 & Norway & - & 51 & 19.6 \\
\hline Schürks et al. [23] & 2012 & Germany & $62.5 \pm 6.3$ & 6857 & 14.5 \\
\hline Sevindik et al. [24] & 2017 & Turkey & $11.36 \pm 3.34$ & 65 & 33.84 \\
\hline van Oosterhout et al. [25] & 2016 & Netherland & $45.1 \pm 11.6$ & 2385 & 16.9 \\
\hline Winter et al. [26] & 2013 & France & $67.3 \pm 8.2$ & 2816 & 8.7 \\
\hline
\end{tabular}

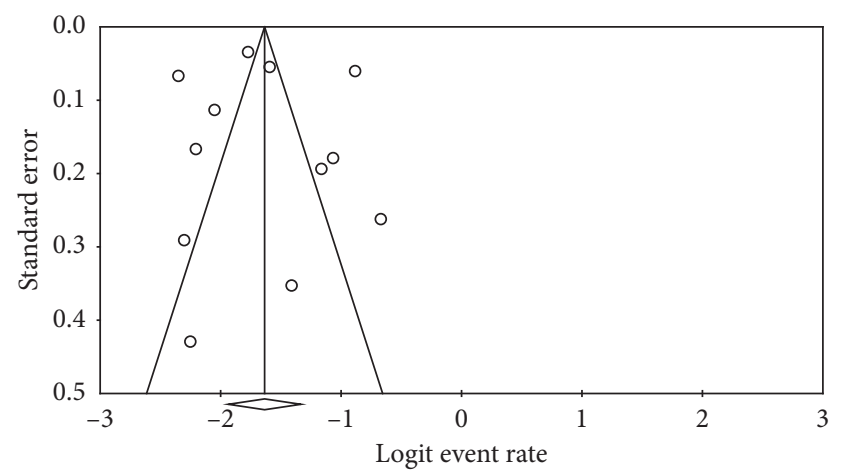

FIGURE 2: Funnel plot of standard error by logit event rate.

the overall prevalence of RLS in patients with migraine was $16.3 \%$ (95\% CI: $12.6-20.8 \%$ )

The prevalence of RLS in patient with migraine was higher in patients $\geq 65$ years of age in Turkey $(33.8 \%$; $95 \%$ CI: $23.4-64.1 \%)$ and, however, was lower $(8.7 \%$; 95\% CI: $7.7-9.8 \%$ ) in France (Figure 3). As illustrated in Figure 3, the prevalence of RLS was based on the random effects model in which the black square is the prevalence rate and the length of the line on which the square is located denotes the confidence interval of $95 \%$ for each study; the overall prevalence for the total studies is shown by the diamond shape.

In order to examine the impacts of potential factors on heterogeneity of RLS in patients with migraine, a metaregression analysis was conducted using the two factors of "sample size" and "year of study" (Figures 4 and 5). According to Figure 4, as sample size increases, the prevalence of restless legs syndrome in patients with migraine decreases, and this was statistically significant $(P<0.05)$. It is also reported in Figure 5 that with increasing the year of study, the prevalence of restless legs syndrome in patients with migraine increases, which was also statistically significant $(P<0.05)$. To explain this, it can be argued that with increasing the sample size and therefore more healthy people entering the study, the incidence of cases in the study of prevalence decreases, and also with the updating of studies and increase in diagnostic methods and their improvement, the prevalence increases.

\section{Discussion}

Epidemiological studies have investigated a variety of migraine-related disorders such as anxiety, depression, and sleep problems among patients [27]. In fact, the World Health Organization (WHO) stated that migraine is a major cause of debility in the general population [18].

Restless legs syndrome/Willis-Ekbom disorder (RLS/ WED) is a movement disorder that most commonly affects the legs and can cause severe sleep disturbance [28]. According to a study conducted by Cho et al., the prevalence of this disorder was estimated to be $2-15 \%$. Moreover, the prevalence increases with age. The International Restless Legs Syndrome Study Group (IRLSSG) provides diagnostic criteria in accordance with clinical examinations and documentation for RLS [18].

The cause of RLS is still unclear; however, dopamine chemicals are believed to be involved in the development of this disorder [29]. According to Suzuki et al., not only the dopaminergic treatments may alleviate the RLS symptoms, but also migraine headaches (if treated with certain therapies such as TCA's and antiepileptic agents such as topiramate) may induce RLS [30]. Medical examinations and history are used to diagnose RLS, and if the disorder progresses, testing for anemia such as iron deficiency is required [29].

According to a study conducted by Fuh et al. the prevalence of RLS in patients with migraine in Western countries is higher than in Asian nations, and this may be related to genetics [12]. RLS, such as migraine, is a neurological disorder. In recent years, the relationship between these two disorders has been investigated in different pieces of research. These two disorders are similar in terms of prevalence in the general population [17].

van Oosterhout et al. reported that the prevalence of RLS in migraineurs is approximately $17 \%$ [25]. Also, according to another study conducted by Schürks et al. on a female population, the prevalence of RLS in migraine patients was reported to be $14.5 \%$ [23]. According to the results of our study and the survey of 15,196 patients, the overall prevalence of RLS in migraineurs is $16.9 \%$.

The most important limitation of this study was the lack of access to the full text of some studies. 


\begin{tabular}{|c|c|c|c|c|c|c|c|c|c|}
\hline \multirow[t]{2}{*}{ Study name } & \multirow[b]{2}{*}{$\begin{array}{c}\text { Event } \\
\text { rate }\end{array}$} & \multicolumn{3}{|c|}{ Statistics for each study } & \multirow[b]{2}{*}{$p$ value } & & \multicolumn{2}{|c|}{ Event rate and 95\% CI } & \\
\hline & & $\begin{array}{l}\text { Lower } \\
\text { limit }\end{array}$ & $\begin{array}{l}\text { Upper } \\
\text { limit }\end{array}$ & $z$ value & & & & & \\
\hline Calhoun et al. [15] & 0.238 & 0.176 & 0.314 & -6.006 & 0.000 & & & & \\
\hline Ødegård Odegard et al. [22] & 0.196 & 0.109 & 0.327 & -4.001 & 0.000 & & & & \\
\hline Chen et al. [16] & 0.114 & 0.093 & 0.138 & -18.107 & 0.000 & & & & \\
\hline Chen et al. [17] & 0.099 & 0.074 & 0.133 & -13.239 & 0.000 & & & & \\
\hline Cho et al. [18] & 0.091 & 0.054 & 0.150 & -7.916 & 0.000 & & & & \\
\hline Cologno et al. [19] & 0.256 & 0.195 & 0.328 & -5.961 & 0.000 & & & & \\
\hline d'Onofrio et al. [20] & 0.095 & 0.043 & 0.196 & -5.245 & 0.000 & & & & \\
\hline Mauyqil et al. [21] & 0.292 & 0.269 & 0.318 & -14.655 & 0.000 & & & & \\
\hline Schürks et al. [23] & 0.145 & 0.137 & 0.154 & -51.712 & 0.000 & & & & \\
\hline Sevindik et al. [24] & 0.338 & 0.234 & 0.461 & -2.557 & 0.011 & & & & \\
\hline van Oosterhout et al. [25] & 0.169 & 0.154 & 0.185 & -29.151 & 0.000 & & & & \\
\hline \multirow[t]{4}{*}{ Winter et al. [26] } & 0.087 & 0.077 & 0.098 & -35.159 & 0.000 & & & & \\
\hline & 0.163 & 0.126 & 0.208 & -10.657 & 0.000 & & & & \\
\hline & & & & & & -1.00 & -0.50 & 0.00 & 1.00 \\
\hline & & & & & & & Favours A & Favours & \\
\hline
\end{tabular}

FIgURE 3: Meta-analysis.

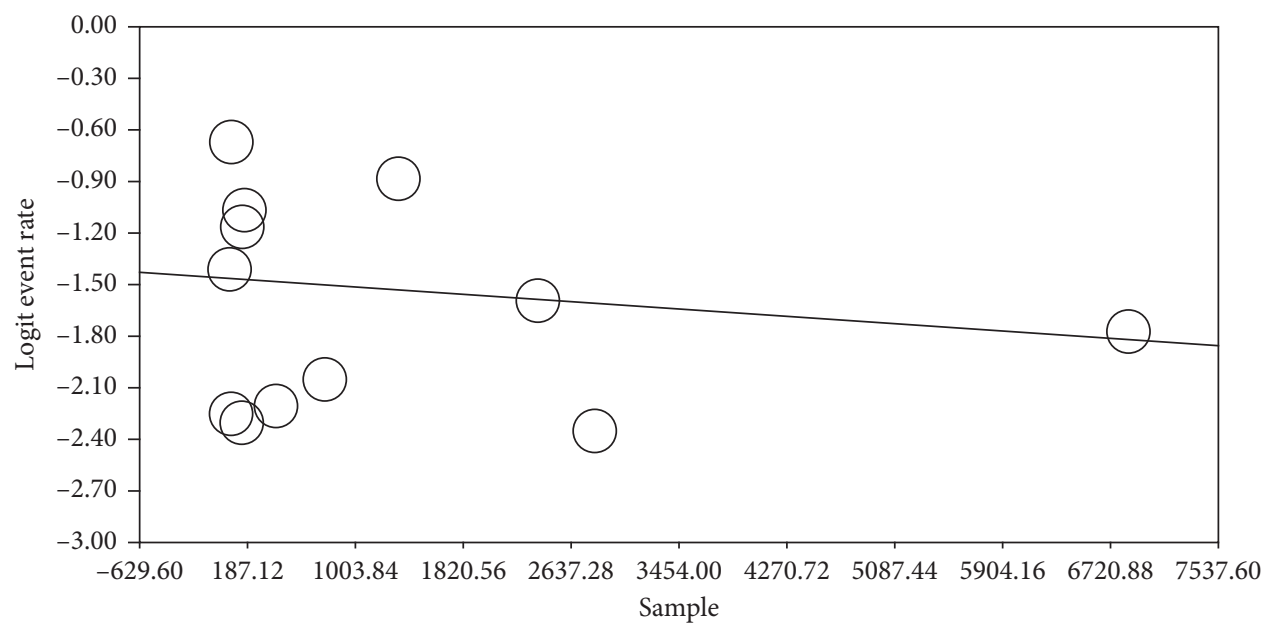

FIgURE 4: Regression of sample on logit event rate.

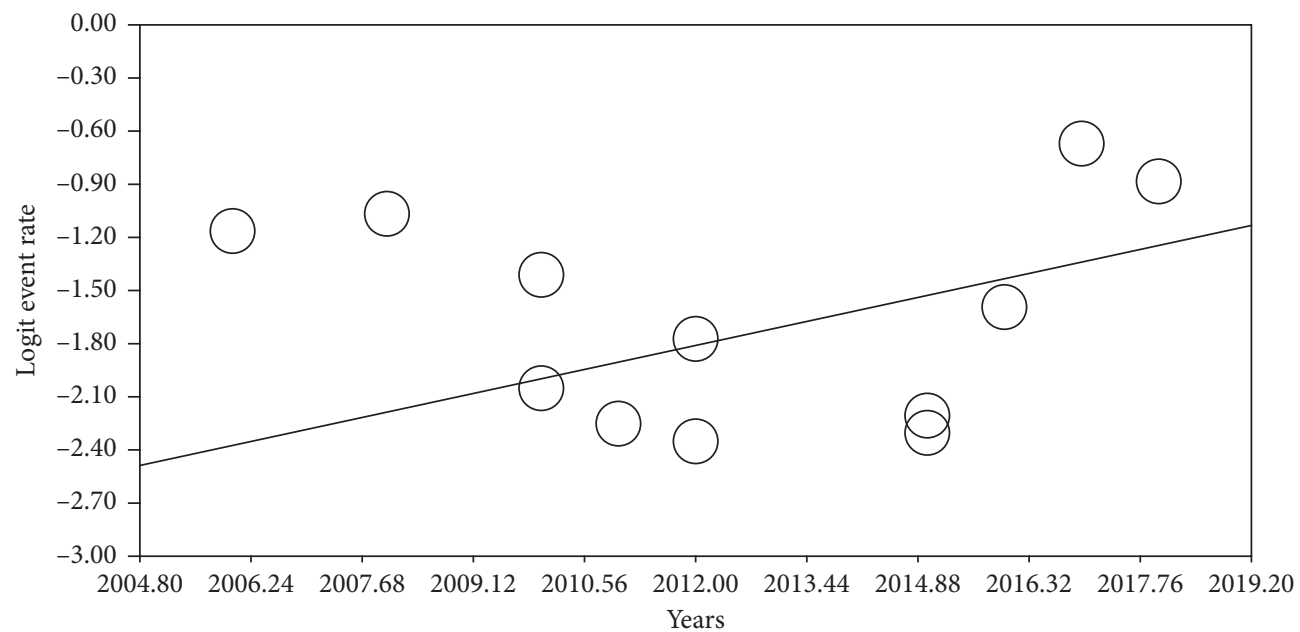

Figure 5: Regression of years on logit event rate. 


\section{Conclusion}

This study showed that RLS is observed in patients with migraine. The results obtained can be used for more focused research works aimed at determining the causes and consequences of RLS in migraine patients. In addition, this study provides a context for further studies on the treatment and impact of different therapies in the RLS population. Future studies may focus on the comparison of the effects of dopaminergic treatments in patients with RLS and migraine.

\section{Abbreviations}

RLS: $\quad$ Restless legs syndrome

SID: $\quad$ Scientific Information Database

STROBE: STrengthening the Reporting of OBservational studies in Epidemiology

PRISMA: Preferred Reporting Items for Systematic Reviews and Meta-Analyses

WHO: World Health Organization

RLS/ Restless legs syndrome/Willis-Ekbom disorder

WED:

IRLSSG: International Restless Legs Syndrome Study Group.

\section{Data Availability}

Datasets are available through the corresponding author upon reasonable request.

\section{Conflicts of Interest}

The authors declare that they have no conflicts of interest.

\section{Authors' Contributions}

HG and BKP contributed to the design, and MM and RJ carried out statistical analysis and participated in most of the study steps. NS and AVR assisted in designing the study and helped in the interpretation of the study. All the authors have read and approved the content of the manuscript.

\section{Acknowledgments}

This study was supported by the Student Research Committee of Kermanshah University of Medical Sciences and Deputy for Research and Technology, Kermanshah University of Medical Sciences (IR) (3009102).

\section{References}

[1] I. Morgan, F. Eguia, B. Gelaye et al., "Sleep disturbances and quality of life in Sub-Saharan African migraineurs," Journal of Headache and Pain, vol. 16, no. 1, p. 18, 2015.

[2] Y. K. Lin, G. Y. Lin, J. T. Lee et al., "Associations between sleep quality and migraine frequency: a cross-sectional case-control study," Medicine, vol. 95, no. 17, Article ID e3554, 2016.

[3] S. Akerman, M. Romero-Reyes, and P. R. Holland, "Current and novel insights into the neurophysiology of migraine and its implications for therapeutics," Pharmacology \& Therapeutics, vol. 172, pp. 151-170, 2017.
[4] C. P. Yang, M. L. Hsieh, J. H. Chiang, H. Y. Chang, and V. C. Hsieh, "Migraine and risk of narcolepsy in children: a nationwide longitudinal study," PLoS One, vol. 12, no. 12, Article ID e0189231, 2017.

[5] J. Berengueres and F. Cadiou, "Migraine factors as reported by smartphone users," in Proceedings of the 2016 38th Annual International Conference of the IEEE Engineering in Medicine and Biology Society (EMBC), vol. 2016, pp. 271-274, Orlando, FL, USA, August 2016.

[6] E. R. De La Torre, R. Quintana, V. Carboni et al., "My migraine voice: a worldwide survey of 11,266 migraine patients," Journal of Headache and Pain, vol. 19, 2018.

[7] X. Wang, H. B. Zhou, J. M. Sun, Y. H. Xing, Y. L. Zhu, and Y. S. Zhao, "The prevalence of migraine in university students: a systematic review and meta-analysis," European Journal of Neurology, vol. 23, no. 3, pp. 464-475, 2016.

[8] B. L. Nye and V. M. Thadani, "Migraine and epilepsy: review of the literature," Headache: The Journal of Head and Face Pain, vol. 55, no. 3, pp. 359-380, 2015.

[9] G. S. Jakobsen, A. M. Timm, A. M. Hansen, A. H. Garde, and K. Nabe-Nielsen, "The association between shift work and treatment-seeking migraine in Denmark," Ergonomics, vol. 60, no. 9, pp. 1207-1217, 2017.

[10] B. A. Acar, T. Acar, A. N. Alagöz et al., "Relationship between primary restless legs syndrome and migraine with aura," The Kaohsiung Journal of Medical Sciences, vol. 32, no. 8, pp. $420-426,2016$.

[11] S. Buturak, D. Tiryaki, E. Dag, and Y. Türkel, "Restless legs syndrome in a bipolar disorder patient treated with low dose olanzapine: is there an association?" Anatolian Journal of Psychiatry, vol. 16, no. 4, pp. 301-303, 2015.

[12] J.-L. Fuh, M.-Y. Chung, S.-C. Yao et al., "Susceptible genes of restless legs syndrome in migraine," Cephalalgia, vol. 36, no. 11, pp. 1028-1037, 2016.

[13] S. Cevoli, G. Giannini, V. Favoni, G. Pierangeli, and P. Cortelli, "Migraine and sleep disorders," Neurological Sciences, vol. 33, no. 1, pp. 43-46, 2012.

[14] M. Fernández-Matarrubia, M. L. Cuadrado, C. M. SánchezBarros et al., "Prevalence of migraine in patients with restless legs syndrome: a case-control study," Headache: The Journal of Head and Face Pain, vol. 54, no. 8, pp. 1337-1346, 2014.

[15] A. H. Calhoun, S. Ford, A. G. Finkel, K. A. Kahn, and J. D. Mann, "The prevalence and spectrum of sleep problems in women with transformed migraine," Headache: The Journal of Head and Face Pain, vol. 46, no. 4, pp. 604-610, 2006.

[16] P. K. Chen, J. L. Fuh, S. P. Chen, and S. J. Wang, "Association between restless legs syndrome and migraine," Journal of Neurology, Neurosurgery \& Psychiatry, vol. 81, no. 5, pp. 524-528, 2010.

[17] P.-K. Chen, J.-L. Fuh, and S.-J. Wang, "Bidirectional triggering association between migraine and restless legs syndrome: a diary study," Cephalalgia, vol. 36, no. 5, pp. 431-436, 2015.

[18] S. J. Cho, Y. K. Chung, J. M. Kim, and M. K. Chu, "Migraine and restless legs syndrome are associated in adults under age fifty but not in adults over fifty: a population-based study," Journal of Headache and Pain, vol. 16, no. 1, p. 75, 2015.

[19] D. Cologno, G. Cicarelli, V. Petretta, F. d'Onofrio, and G. Bussone, "High prevalence of dopaminergic premonitory symptoms in migraine patients with restless legs syndrome: a pathogenetic link?" Neurological Sciences, vol. 29, no. 1, pp. $166-168,2008$.

[20] F. d'Onofrio, D. Cologno, V. Petretta et al., "Restless legs syndrome is not associated with migraine with aura: a clinical 
study," Neurological Sciences, vol. 32, no. S1, pp. 153-156, 2011.

[21] T. Muayqil, B. N. Al-Jafen, Z. Al-Saaran et al., "Migraine and headache prevalence and associated comorbidities in a large Saudi sample," European Neurology, vol. 79, no. 3-4, pp. 126-134, 2018.

[22] S. S. Ødegård, M. Engstrøm, T. Sand, L. J. Stovner, J.-A. Zwart, and K. Hagen, "Associations between sleep disturbance and primary headaches: the third Nord-Trøndelag health study," The Journal of Headache and Pain, vol. 11, no. 3, pp. 197-206, 2010.

[23] M. Schürks, A. C. Winter, K. Berger, J. E. Buring, and T. Kurth, "Migraine and restless legs syndrome in women," Cephalalgia, vol. 32, no. 5, pp. 382-389, 2012.

[24] M. S. Sevindik, S. Demirci, B. Göksan et al., "Accompanying migrainous features in pediatric migraine patients with restless legs syndrome," Neurological Sciences, vol. 38, no. 9, pp. 1677-1681, 2017.

[25] W. P. J. van Oosterhout, E. J. W. van Someren, M. A. Louter et al., "Restless legs syndrome in migraine patients: prevalence and severity," European Journal of Neurology, vol. 23, no. 6, pp. 1110-1116, 2016.

[26] A. C. Winter, M. Schürks, K. Berger, J. E. Buring, J. M. Gaziano, and T. Kurth, "Migraine and restless legs syndrome in men," Cephalalgia, vol. 33, no. 2, pp. 130-135, 2013.

[27] Z. Zhu, X. Fan, X. Li, G. Tan, L. Chen, and J. Zhou, "Prevalence and predictive factors for poor sleep quality among migraineurs in a tertiary hospital headache clinic," Acta Neurologica Belgica, vol. 113, no. 3, pp. 229-235, 2013.

[28] L. M. Trotti, "Restless legs syndrome and sleep-related movement disorders," Continuum: Lifelong Learning in Neurology, vol. 23, no. 4, pp. 1005-1016, 2017.

[29] C. C. Muth, "Restless legs syndrome," JAMA, vol. 317, no. 7, p. 780, 2017.

[30] K. Suzuki, S. Suzuki, M. Miyamoto et al., "Does pramipexole treatment improve headache in patients with concomitant migraine and restless legs syndrome?" Tremor and Other Hyperkinetic Movements, vol. 3, p. 3, 2013. 